\begin{tabular}{cc} 
Sharif University of Technology \\
Scientia Iranica \\
SCIENTIA & Transactions B: Mechanical Engineering \\
I RAN I CA & \\
\hline
\end{tabular}

\title{
Vapor solidification of saturated air in two-dimensional stagnation flow
}

\author{
A. Shokrgozar Abbasi* and M. Ghayeni \\ Department of Mechanical Engineering, Payame Noor University, Mashhad, Iran.
}

Received 5 August 2017; received in revised form 5 November 2018; accepted 10 December 2018

\author{
KEYWORDS \\ Water vapor \\ solidification; \\ Stagnation flow; \\ Unsteady flow; \\ Viscous fluid; \\ Incompressible flow; \\ Similarity solution.
}

\begin{abstract}
This study aims to investigate the stagnation-flow solidification of vapor from saturated air. Saturated air with strain rate $a$ impinges on a flat plate and, thus, condensation occurs and an icy layer forms on the plate because the plate temperature is below the freezing temperature of water. The ice surface was modeled as an accelerated flat plate that moves toward the impinging fluid. The unsteady Navier-Stokes equations were subjected to a similarity transformation to obtain a single ordinary differential equation for velocity distribution. Two methods of solution were used for the energy equation: a finitedifference numerical technique and a numerical solution of a similarity equation. These two results were compared to determine the superior accuracy. First, freezing time increases as the far-field temperature decreases from $0^{\circ} \mathrm{C}$ and, then, rapidly approaches zero as the farfield temperature approaches $0^{\circ} \mathrm{C}$. Despite the expectation that condensation would begin at the substrate in a physical experiment, here, the size of the cell next to the substrate controls the time at which condensation begins. It was found that the maximum time before freezing began at an air temperature of about $5^{\circ} \mathrm{C}$ for 0.1 and $0.2 \mathrm{~mm}$ sizes. The ultimate frozen thickness for two saturated air temperatures was presented.
\end{abstract}

(C) 2020 Sharif University of Technology. All rights reserved.

\section{Introduction}

In the present study, the freezing of water vapor from saturated air is investigated in two-dimensional Cartesian stagnation flow. Solidification is one of the most interesting phenomena in natural processes and industrial applications. This phenomenon comprises heat transfer accompanied by phase change. Glass, metal, plastic and oil, food, and other corresponding industries require good insight into solidification behavior as the nature of solid growth. Similarly, studies relating to phase change in stagnant media can help

\footnotetext{
*. Corresponding author. Tel.: +985138683887; Fax: +98 5138451620

E-mailaddress: shokrgozar.ali@gmail.com (A. Shokrgozar Abbasi)
}

doi: $10.24200 /$ sci. 2018.4956.1007 acquire a better understanding of the convection effect upon interface behavior. In the aerospace industry, the freezing of saturated air vapor and the conditions for its freezing in front of the aircraft is very important. This phenomenon may also occur at the tip of a missile. Investigations in the field of heat transfer in phase change or solidification in stagnation flow with or without similarity solutions and related studies are presented in the following. The classic problem of ice formation in polar seas was solved using the analytical method by Stefan [1]. The one-dimensional heat flux for phase change issues was presented by Goodrich [2]. An experimental study on natural convection in the fluid border between liquid and solid phases was carried out by Sparrow et al. [3]. Numerical methods for solving the problems of freezing the flow of natural convection between two isolated plates were presented by Lacroix [4]. A three-dimensional numerical study of free convection with phase change in a channel 
with a rectangular cross-section was performed by Yeoh et al. [5]. An analysis of the combination of hydrodynamic behavior and behavior of the solidliquid boundary layer of fluids, located between two freezing isolated plates, was carried out by Hadji and Schell [6]. A method for calculating time-dependent heat flux caused by natural convection during freezing fluid between two isolated plates was presented by Hanumanth [7]. An integrated model for continuous phase change issues was presented and investigated by Oldenburg and Spera [8]. The problem of freezing molten liquid drops on a rigid plate model was solved by Trapaga et al. [9], and a comparison between numerical modeling and laboratory results of deformation and solidification of a drop on a cold plate was made by Watanabe et al. [10]. Model transformation and freezing adjusted to a drop impinging on the substrate plate was evaluated by Marchi et al. [11]. The flow and heat transfer of viscous fluid was produced by the axisymmetric stagnation flow on a flat plate whose coordinates damped oscillatory motion, as investigated by Weidman and Mahalingam [12]. Flow and heat transfer in the asymmetric threedimensional viscous stagnation flow was investigated by Shokrgozar and Rahimi [13]. Viscous stagnation flow and heat transfer in the asymmetric three-dimensional system with suction and blowing was considered by Shokrgozar and Rahimi [14]. The stagnation-point flow of Walters-B fluid induced by a Riga plate was investigated by Shafiq et al. [15]. The problem of a steady Magnetohydrodynamics (MHD) boundary layer flow over a semi-infinite stretching surface with a power-law velocity was considered by Hammouch et al. [16]. A hygro-thermo-mechanical multiphase model that describes the freezing behavior of partially saturated air-entrained concrete was investigated by Eriksson et al. [17]. The unsteady stagnation-point boundary layer flow and heat transfer of a particular third-grade fluid past a permeable stretching/shrinking sheet was studied by Naganthran et al. [18]. The impact of the melting phenomenon in the magnetohydrodynamic stagnation-point flow of nanofluid on a nonlinear stretching surface with variable thicknesses was considered by Farooq et al. [19]. The simultaneous characteristics of thermal radiation and melting heat transfer effects in the stagnation-point flow of carbon nanotubes due to a stretching cylinder were investigated by Hayat et al. [20]. The exact solution of the unsteady two-dimensional stagnation flow and heat transfer on a heated plate was presented by Shokrgozar and Rahimi [21]. The two-dimensional stagnation flow and heat transfer on an accelerated flat plate was investigated by Shokrgozar and Rahimi [22]. Axisymmetric stagnation flow and heat transfer on an accelerated flat plate was presented by Shokrgozar et al. [23]. Stagnation-flow solidification of an inviscid fluid that freezes at a common border was considered by Brattkus and Davis [24]. The problem of Stephen's solidification of inviscid fluid in stagnation flow was solved by Rangel and Bian [25]. Freezing at the subcooled liquid stagnation point (freezing point) was investigated in the two-dimensional Cartesian coordinate system by Lambert and Rangel [26]. Viscous fluid phase change in stagnation flow was considered by Joo-Sik [27]. The steady-state, viscous flow and heat transfer of nano-fluid in the vicinity of an axisymmetric stagnation point of a stationary cylinder with constant wall heat flux was investigated by Mohammadiun et al. [28]. The unsteady three-dimensional axisymmetric stagnation-point flow of a viscous compressible fluid on a flat plate was investigated by Rahimi and Mozayyeni [29]. The steady-state viscous flow and heat transfer in the vicinity of a non-axisymmetric stagnation point of an infinite stationary cylinder was investigated by Alizadeh et al. [30]. Solidification of incompressible fluid in the two-dimensional stagnation flow was considered by Shokrgozar and Rahimi [31]. The freezing of incompressible fluid in unsteady axisymmetric stagnation flow was also investigated by Shokrgozar [32]; however, the solidification of vapor from saturated gas has not been investigated so far. In this study, saturated air vapor freezing in stagnation flow is closely analyzed. Fluid flow is assumed to be incompressible and viscous in the laminar regime and, also, long in $y$ direction. Due to the fluid contact with a plate at a temperature lower than the temperature of water, the vapor becomes liquid and, then, if the substrate temperature is low enough, ice will form. Note that some subcooling is usually required for the condensation of vapor from gas to occur. The same applies to the freezing of liquid water to ice. Here, if a high rate of heat transfer and adequate time to settle at the lowest node are not provided, distilled water may turn into frost. This paper assumes that there is adequate time available for the fluid to settle so that the frost formation assumption can be safely neglected; thus, frost formation requires a separate investigation. Since considered velocities are low in this study, inertia and Magnus forces due to their rotation in response to shear are assumed negligible. The Magnus forces are assumed negligible in this paper due to their rotation in response to shear. The effects of mass diffusion are also neglected for the sake of simplicity.

\section{Problem formulation}

In this study, the flow in $y$ direction is so long that it is assumed to be two-dimensional. In Figure 1, twodimensional Cartesian coordinates with corresponding velocities $(u, w)$ with respect to $(x, z)$ are shown. Saturated air stagnation flow with strain $a(t)$ in $z$ direction approaches $z=0$, perpendicular to the plate. The 


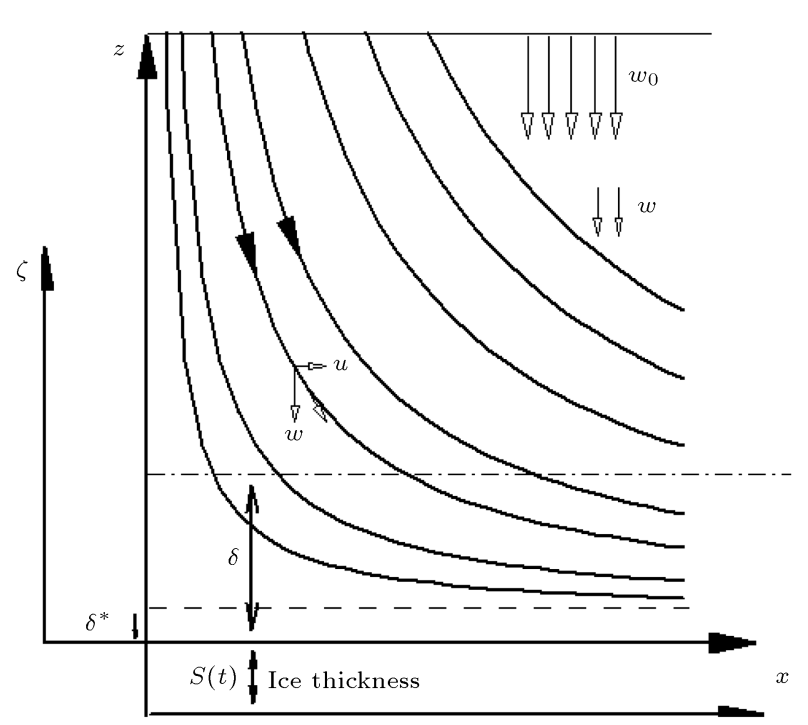

Figure 1. Two-dimensional solidification in stagnation flow (problem schematic diagram).

flow is assumed to be laminar with constant properties for air, vapor, and water. If the plate temperature is sufficiently low, condensation occurs and the resulting liquid freezes. The ice surface is modeled as an accelerated flat plate moving toward an impinging fluid with variable velocity $\dot{S}(t)$ and acceleration $\ddot{S}(t)$, respectively, by the distance $S(t)$ in each time step. For more information on the substitution of an imaginary flat plate at the solid-fluid interface, please refer to Shokrgozar and Rahimi [31].

Due to the temperature change in the air, densities of vapor and air vary. These changes will be negligible, as the flow is assumed incompressible. However, the change in the volume conversion of vapor to water, which affects cell size in the mesh, has been taken into account. In this situation, there are particles of water and vapor together; thus, the fluid has a multiphase state. However, the mass of water particles is too small to destroy homogeneity. Therefore, the multiphase effects are neglected in equations. However, the same small mass of condensation is important in the energy equation. Note that the fine particles of water present only in the lowest node have an opportunity to settle as the lowest velocity and the highest water weight in this row. Higher water weights in the lowest node result from the increase and accumulation of fine particles on each other. Otherwise, water vapor in other nodes does have an opportunity to settle and, then, frost will form in this mode, which is outside the scope of this article and requires separate investigations. Mass and Navier-Stokes equations for Newtonian, laminar, incompressible, unsteady states with constant density and viscosity properties of the fluid provide the following:

Mass:

$$
\frac{\partial u}{\partial x}+\frac{\partial w}{\partial z}=0
$$

Momentum:

$$
\begin{aligned}
& \frac{\partial u}{\partial t}+u \frac{\partial u}{\partial x}+w \frac{\partial u}{\partial z}=-\frac{1}{\rho} \frac{\partial p}{\partial x}+\nu\left(\frac{\partial^{2} u}{\partial x^{2}}+\frac{\partial^{2} u}{\partial z^{2}}\right) \\
& \frac{\partial w}{\partial t}+u \frac{\partial w}{\partial x}+w \frac{\partial w}{\partial z}=-\frac{1}{\rho} \frac{\partial p}{\partial z}+\nu\left(\frac{\partial^{2} w}{\partial x^{2}}+\frac{\partial^{2} w}{\partial z^{2}}\right)
\end{aligned}
$$

The Magnus forces due to their rotation in response to shear are assumed to be negligible. Note that the small mass of water is indeed a homogeneous mixture in saturated air, and the continuous formula of partial derivatives is used with respect to time. In addition, the effects of mass diffusion are neglected for simplicity. Unsteady energy equation in the fluid region (dissipation and radiation heat transfer are neglected without internal source) gives:

$$
\begin{aligned}
\frac{\partial T}{\partial t} & +u \frac{\partial T}{\partial x}+w \frac{\partial T}{\partial z}=\alpha\left(\frac{\partial^{2} T}{\partial x^{2}}+\frac{\partial^{2} T}{\partial z^{2}}\right) \\
& +\frac{\partial m_{v l}}{\partial t} \frac{H_{v l}}{\sum_{j=1}^{3} m_{j} C_{j}} .
\end{aligned}
$$

Note that conductivity and heat capacity coefficients are constant ( $k$ and $C$, respectively). Moreover, $d u \approx$ $c d T$ is assumed where $p, \rho, \nu$, and $\alpha$ denote the fluid pressure, density, kinematic viscosity, and thermal diffusivity, respectively. follows:

Energy equation in the solid phase (ice) is as

$$
m C \frac{\partial T}{\partial t}=k_{S} \frac{\partial T}{\partial S} A_{S}-k_{l} \frac{\partial T}{\partial N} A_{N} .
$$

Moreover, the energy equation (boundary condition) at the interface before reaching freezing temperature becomes:

$$
\begin{aligned}
& \sum_{j=1}^{3} \rho_{j} C_{j} \forall_{j} \frac{\partial T}{\partial t}=k_{S} \frac{\partial T}{\partial S} A_{S}-k_{l} \frac{\partial T}{\partial N} A_{N} \\
& +\left(\frac{m_{v}^{n}-m_{v}^{n+1}}{\Delta t}\right) H_{v l},
\end{aligned}
$$

where the term on the left in the equation is energy moving boundary and those on the right side are conductive heat transfers and heat source from left to right, respectively. Furthermore, the energy equation (boundary condition) at the interface after reaching freezing temperature becomes:

$$
\rho_{s} H_{l s} \frac{\partial S}{\partial t}=k_{S} \frac{\partial T}{\partial S}-k_{l} \frac{\partial T}{\partial N} .
$$


Here, the left term is the source and the right ones are conductive heat transfers from up and down, respectively.

Similarly, by applying curve fitting, the humidity ratio from the psychometric chart can be defined as follows:

$$
\begin{aligned}
\omega_{i, k}^{n+1}= & b_{1}+b_{2} T_{i, k}^{n+1}+b_{3}\left(T_{i, k}^{n+1}\right)^{2}+b_{4}\left(T_{i, k}^{n+1}\right)^{3} \\
& +b_{5}\left(T_{i, k}^{n+1}\right)^{4} .
\end{aligned}
$$

Eq. (8) can be used in the range of 0 to 70 degrees in Celsius that produces the following equation after linearizing through the Newton-Raphson method:

$$
\begin{aligned}
\omega_{i, k}^{n+1}= & \omega_{i, k}^{n}+\left(b_{2}+2 b_{3} T_{i, k}^{n}+3 b_{4}\left(T_{i, k}^{n}\right)^{2}\right. \\
& \left.+4 b_{5}\left(T_{i, k}^{n}\right)^{3}\right)\left(T_{i, k}^{n+1}-T_{i, k}^{n}\right)
\end{aligned}
$$

where subscripts $(i, k)$ are node numbers in $x$ and $z$ directions, respectively; superscript $(n+1)$ is a new time, $\omega_{i, k}^{n+1}$ is the humidity ratio for new temperature, and $b\left({ }^{\circ} \mathrm{C}\right)^{0}$ to ${ }^{-4}$ coefficients include the following:

$$
\begin{aligned}
& b_{1}=3.744 \mathrm{E}-03\left({ }^{\circ} \mathrm{C}\right)^{0}, \\
& b_{2}=2.820 \mathrm{E}-04\left({ }^{\circ} \mathrm{C}\right)^{-1}, \\
& b_{3}=7.360 \mathrm{E}-06\left({ }^{\circ} \mathrm{C}\right)^{-2}, \\
& b_{4}=2.200 \mathrm{E}-07\left({ }^{\circ} \mathrm{C}\right)^{-3}, \\
& b_{5}=3.270 \mathrm{E}-09\left({ }^{\circ} \mathrm{C}\right)^{-4} .
\end{aligned}
$$

Note that the curve fitting method is used to find the coefficients, and Eq. (9) is obtained for linearizing the numerical solution of the energy equation.

\section{Similarity solution}

\subsection{Fluid flow solution}

According to [16] and integrating Eqs. (1)-(3) outside the boundary layer, the classical equations of potential flow solution are as follows:

$$
U=a(t) x \text {. }
$$

Note that the boundary layer is defined here as the edge of the points, where their velocity is $99 \%$ of their corresponding potential velocity. By substituting Eq. (10) in the continuity equation (Eq. (1)) and integrating them, we have:

$$
W=-a(t) \zeta
$$

where $\zeta=z-S(t)$, and $S(t)$ is the amount of plate displacement in $z$ direction and is assumed to be positive when the plate moves toward the impinging flow. Hence, $S(t)$ and $\zeta$ are the functions of time. The recent Eqs. (10)-(11) are the boundary condition equations in the viscous layer. The reduction of the Navier-Stokes equations is achieved by the following coordinate separation, in which the solution of the viscous problem inside the boundary layer is obtained by composing inviscid and viscous parts of the velocity:

$$
\begin{aligned}
& u=a(t) x f^{\prime}(\eta), \\
& w=-\sqrt{\nu / a_{0}} a(t) f(\eta), \\
& \eta=\sqrt{\nu / a_{0}} \zeta,
\end{aligned}
$$

where the terms involving $f(\eta)$ in Eqs. (12)-(13) denote the similarity form for unsteady stagnation-point flow, and prime represents differentiation with respect to $\eta$. Moreover, $a_{0}$ is the reference potential flow strain rate at the outset of the specified time period. The insertion of the transformations (Eqs. (12)-(14)) into Eqs. (2)(3) yields an ordinary differential equation in terms of $f(\eta)$ :

$$
f^{\prime \prime \prime}+f^{\prime \prime}(\tilde{\dot{S}}+\tilde{a} f)+\left(-\tilde{a} f^{\prime}-\frac{1}{\tilde{a}} \frac{d \tilde{a}}{d \tau}\right) f^{\prime}-\frac{1}{\tilde{a} \xi} \frac{d \tilde{p}}{d \xi}=0
$$

where:

$$
-\frac{1}{\tilde{a} \xi} \frac{d \tilde{p}}{d \xi}=\frac{1}{\tilde{a}} \frac{d \tilde{a}}{d \tau}+\tilde{a},
$$

with Boundary conditions:

$$
\eta=0: \quad f=0, \quad f^{\prime}=0, \quad \eta \rightarrow \infty: \quad f^{\prime}=1 .
$$

Note that in Eqs. (2) and (3), $\frac{\partial u}{\partial t}$ terms are not taken into account since the flow is assumed fully developed; however, since the parameters $\tilde{a}, \frac{d \tilde{a}}{d \tau}$, and $\dot{S}$ are time-dependent, Eq. (15) is also dependent on time. The ordinary differential equation (Eq. (15)) was solved numerically using a shooting method (trial and error) based on the Runge-Kutta algorithm. For more information, refer to Shokrgozar and Rahimi [16].

\subsection{Heat transfer solution}

Dimensionless temperature is defined as follows:

$$
\theta=\frac{T(\eta)-T_{\text {sub }}}{T_{\infty}-T_{\text {sub }}}
$$

By making use of transformations (Eqs. (12)-(14)), the energy equation (Eq. (4)) may be written as follows:

$$
\theta^{\prime \prime}+\operatorname{Pr} \theta^{\prime}\left[f+\tilde{\dot{S}}+\tilde{\dot{S}}_{v l} E v a p\right]=0
$$

where:

$$
\text { Evap }=-\frac{\dot{m}_{\mathrm{air}} H_{v l}}{\sum_{j=1}^{3} m_{j} C_{j}}\left(b_{2}+2 b_{3} \theta+3 b_{4} \theta^{2}+4 b_{5} \theta^{3}\right) \text {. }
$$


Here, the dimensionless water vapor condensing velocity, $\stackrel{\dot{S}}{v l}_{\text {, }}$ is defined as follows:

$$
\tilde{\dot{S}}_{v l}=\tilde{\dot{S}} \times \frac{\rho_{\text {Water }}}{\rho_{\text {Vapor }}},
$$

with boundary conditions as:

$$
\eta=0: \quad \theta=0, \quad \eta \rightarrow \infty: \quad \theta=1,
$$

where $\theta$ is the dimensionless temperature; subscripts $s u b$ and $\infty$ refer to the conditions at the substrate and in the free stream, respectively, $\operatorname{Pr}=\frac{\nu}{\alpha}$ is Prandtl number and prime indicates differentiation with respect to $\eta$. Again, the left term in Eq. (4), $\frac{\partial T}{\partial t}$, is not taken into account, however since $u$ and $v$ velocities are timedependent, Eq. (18) is also dependent on time.

\section{Solution approaches}

The momentum equation (Eq. (15)) is solved numerically using a shooting method (trial and error) based on the fourth-order Runge-Kutta algorithm. The velocity results are used in the energy equation (Eq. (4)) upon the fluid region to convert this nonlinear equation into an ordinary equation (Eq. (18)). The energy equation solution approach to the fluid region is divided into two parts: exact solution and numerical solution. In fact, the exact solution of the energy equation is the quasisteady solution of the heat transfer equation and does not provide temperature profiles for all unsteady times; however, it is used to evaluate the numerical solution. In a numerical solution, to solve the algebraic system of equations, TDMA (Three Diagonal Matrix Algorithm) within ADI (Alternating Direction Implicit) method is used. The energy equation at the solid-fluid interface is divided into two situations: a solution before reaching the freezing point temperature (Eq. (6)) and the one after Eq. (7). In the first situation, the quantities of energy equation terms are dependent on relative humidity. However, the relationship between humidity ratio and temperature (Eq. (8)) is strongly nonlinear. The Newton-Raphson method for linearization is used in the case of relative humidity, which transforms the equation to linear one (Eq. (9)). In the second situation, the energy required for freezing the water is very smaller than that for the condensing latent enthalpy of vapor to water (about $1 / 7.5$ times smaller). Thus, a large under-relaxation factor is necessary for the latent enthalpy term in the energy equation to balance the energy between cells for preventing the responses from divergence. While the method used for solving the problem is implicit, trial and error is required with very small steps due to strong nonlinearity. When the fluid temperature near the ice reaches the freezing point, the temperature of the solid-fluid interface remains constant; as a result, the temperature changes of the fluid region are halted and the energy equation at the solid-fluid interface becomes the same moving solidification boundary condition (Eq. (7)). The solution approaches of the solid-fluid interface equations are numerical. All these equations are solved in every step, simultaneously. Since variations in the size of the mesh from 0.2 to $0.1 \mathrm{~mm}$ produce a negligible variation in the curve, $T_{s u b, \max }$, it can be concluded that the mesh size of $0.2 \mathrm{~mm}$ leads to relatively accurate results.

\section{Validation}

The exact solution of momentum equations does not require validation. However, in this section, the numerical solution results of the energy equation are compared to the exact solution results of this equation. In fact, the exact solution of the energy equation is a quasi-steady solution to the heat transfer equation and does not provide temperature profiles at all unsteady times; however, it is used to evaluate the numerical solution in the final time durations of each step, where the instantaneous temperature profile goes close to the steady state. For a better comparison, the parameter introduction in numerical and exact solutions is the same. Therefore, closing the temperature profiles together means validation of the numerical solution. The results of these comparisons are presented in the next section.

\section{Results}

In Figure 2, dimensionless velocity components $\bar{u}$ and $\bar{w}$ in $x, z$ directions are shown, respectively; however, the velocities obtained in the solution of the energy equation are dimensional.

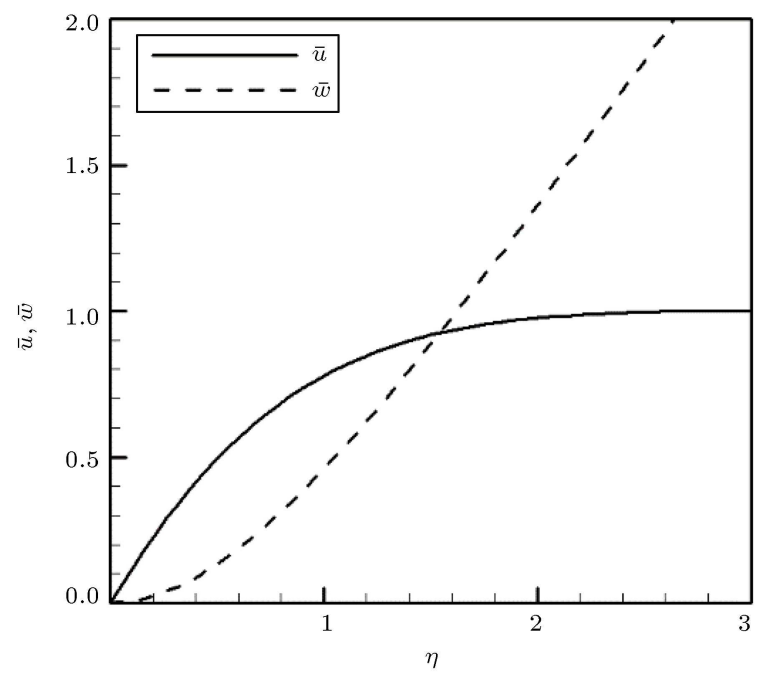

Figure 2. Dimensionless velocity component $\bar{u}$ and $\bar{w}$ profiles in $x$ and $z$ directions, respectively. 
The transformation of the fluid temperature profile to a steady state profile is shown in Figure 3 at a far-field air temperature of $20^{\circ} \mathrm{C}$. For an infinity air temperature to reach the freezing point, the profile changes and, once the temperature arrives at the freezing point of water (zero degrees), the thermal profile becomes constant and will remain unchanged until an ice layer forms.

As mentioned earlier, in order to validate the obtained heat transfer results, the exact solution of energy equation (Eq. (18)) is used. Nevertheless, the results of the exact solution are useful only for the final time durations in each step close to the steadystate condition. In Figure 4, the numerical and exact solutions of energy equations are compared together at

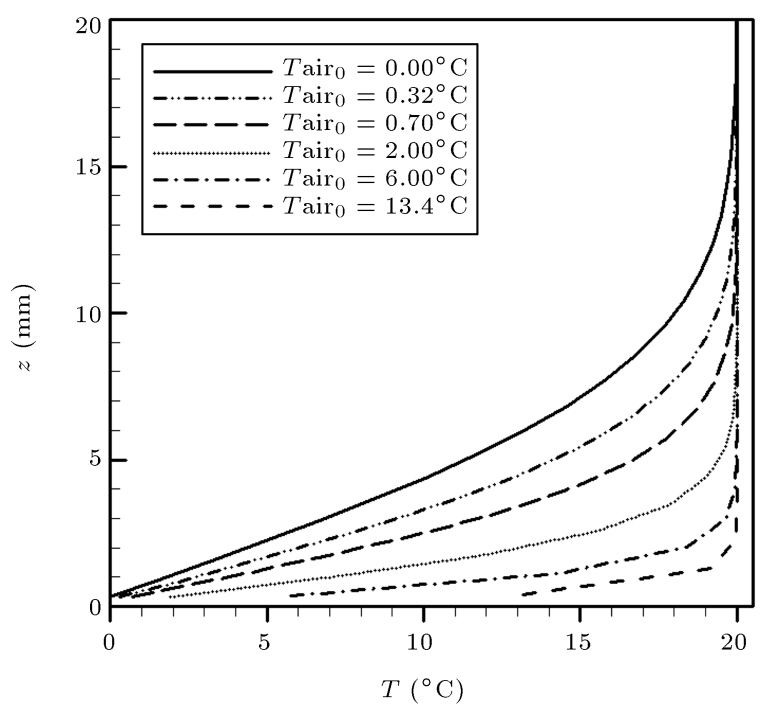

Figure 3. Temperature changing from unsteady to steady for $\operatorname{Tair}_{\infty}=20^{\circ} \mathrm{C}\left(\right.$ Tair $_{0}$ means $T$ air at the nearest cells to ice).

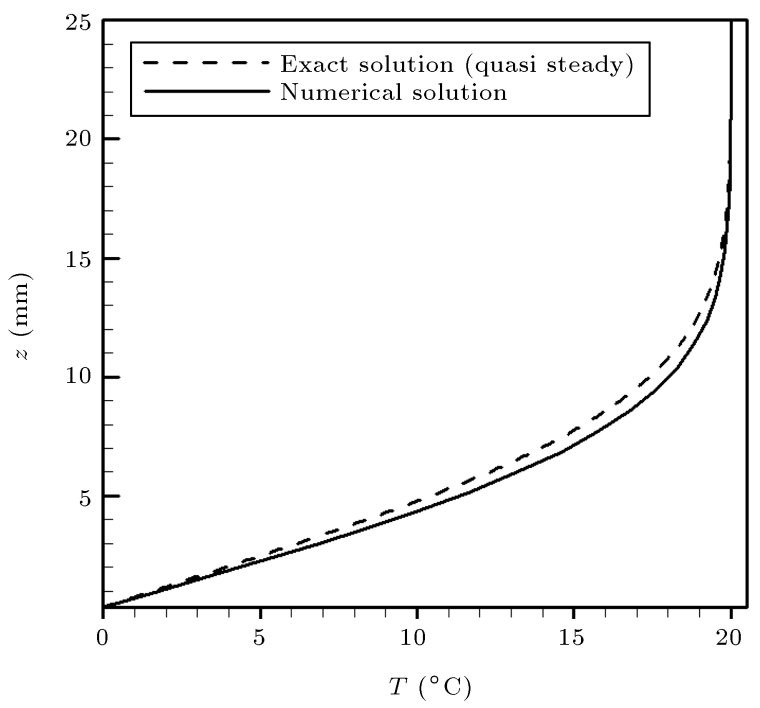

Figure 4. Comparison of the temperature profiles of numerical and exact solutions $\left(\operatorname{Tair}_{\infty}=20^{\circ} \mathrm{C}\right)$. a far-field air temperature of $T$ air $_{\infty}=20^{\circ} \mathrm{C}$. Here, good agreement between these two diagrams is observed. Note that the numerical solution data are at the end time of the steady state. Small differences between the values of numerical and exact solutions result from linearization in the numerical solution in the humidity ratio term and the accumulation of rounding errors.

Figure 5 shows a comparison between the reduction of the vapor mass of saturated air versus that of its temperature and the increase of the water in the cell at a far-field temperature of Tair $_{\infty}=20^{\circ} \mathrm{C}$. As expected, the cell vapor mass at temperature zero approaches zero degree and, thus, does not reach zero; however, at a temperature point of $20^{\circ} \mathrm{C}$, vapor begins to condensate, which is a sign of saturation. Note that the amount of vapor in the air never exactly equals zero. In Figure 6, the humidity ratio with temperature changes is also shown.

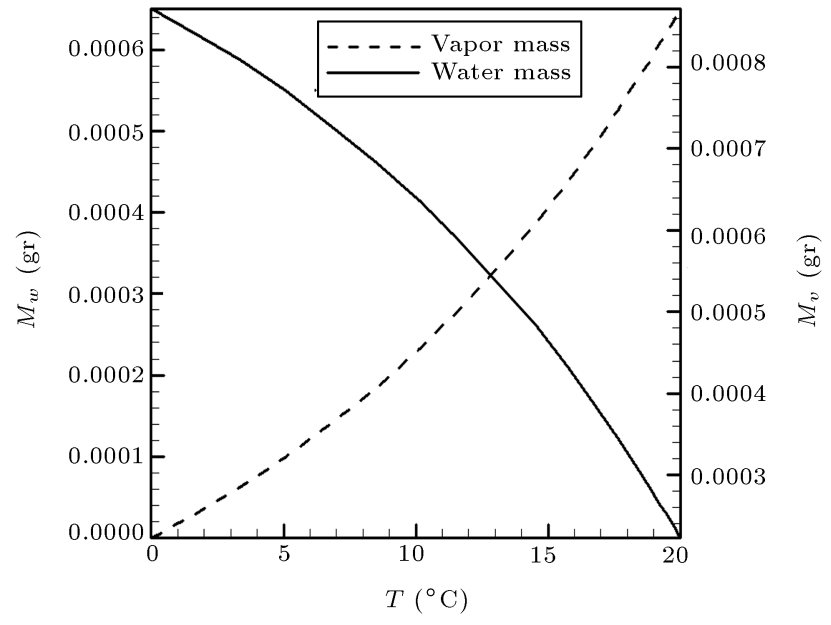

Figure 5. Variations in vapor and water masses in the cells for far-field saturated air $\operatorname{Tair}_{\infty}=20^{\circ} \mathrm{C}$.

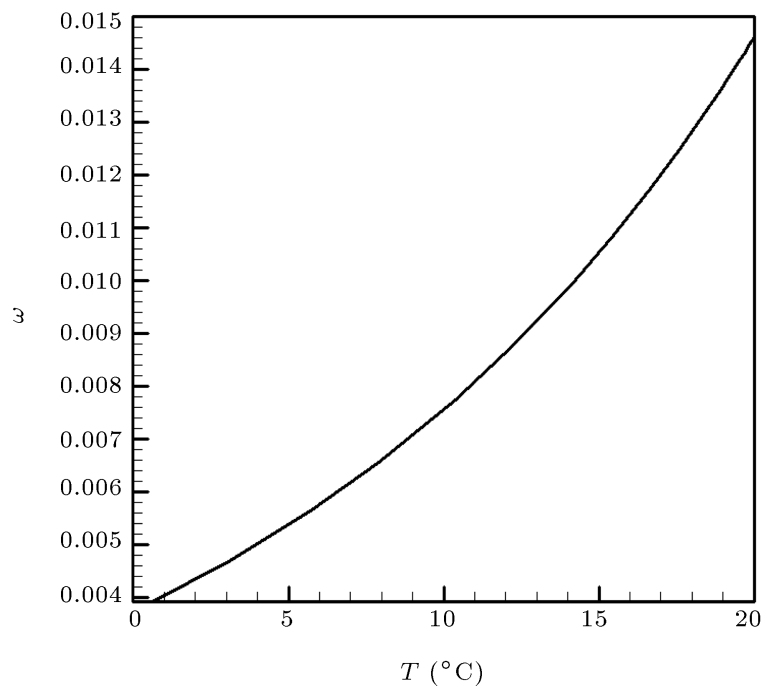

Figure 6. Humidity ratio versus air temperature for far-field saturated air at $\operatorname{Tair}_{\infty}=20^{\circ} \mathrm{C}$. 


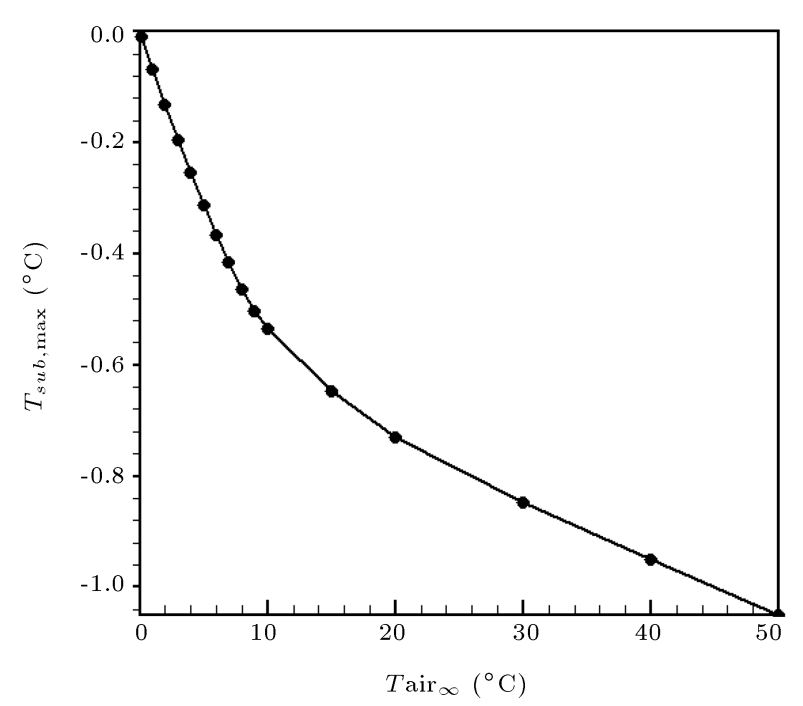

Figure 7. Maximum temperature of the substrate to start freezing versus the far-field air temperature.

One of the most important results in this paper is shown in Figure 7, where the substrate temperatures needed to initiate freezing are provided at different farfield air temperatures. For example, at a far-field air temperature of $\operatorname{Tair}_{\infty}=5^{\circ} \mathrm{C}$, the maximum substrate temperature should be lower than $T_{s u b, \text { max }}=-0.32^{\circ} \mathrm{C}$ to start freezing and, at a far-field air temperature of Tair $_{\infty}=20^{\circ} \mathrm{C}$, the substrate temperature should be lower than $T_{s u b, \text { max }}=-0.80^{\circ} \mathrm{C}$. However, the substrate temperature approaches zero degree with a decrease in the far-field temperature while decreasing water vapor mass in the air. This behavior shown in Figure 7 is generally plausible, as suggested by the simple analysis that follows. Herein, this study assumes that the viscous layer thickness is $\delta$ and $y$ is the distance from the substrate to the gas. The temperature, $T$, profile in the viscous layer is simply represented as follows:

$$
\frac{T-T_{s u b, \max }}{T_{\infty}-T_{s u b, \max }}=\frac{1}{f} \frac{y}{\delta},
$$

where $f$ is a variable factor. Since the amount of vapor in saturated gas roughly doubles for each $15^{\circ} \mathrm{C}$ temperature increase, a variable factor, $f$, between $1 / 8-1 / 3$ is plausible. The value of interest, $y$, can be taken as a fraction, $1 / N$, of the thermal viscous layer thickness; for a location in the middle of the cell next to the substrate $N=150$ where the computational cell size is $1 / 75$ times the thermal viscous layer thickness. To reach the freezing point at the computational node closest to the substrate, Eq. (22) gives:

$$
T_{s u b, \max }=[f N /(f N-1)] T-T_{\infty} /(f N-1) .
$$

For $0^{\circ} \mathrm{C}$ subcooling of the vapor $\left(T=0^{\circ} \mathrm{C}\right), \mathrm{Eq} .(23)$ gives:

$$
T_{\text {sub, } \max }=-T_{\infty} /(150 f-1) .
$$

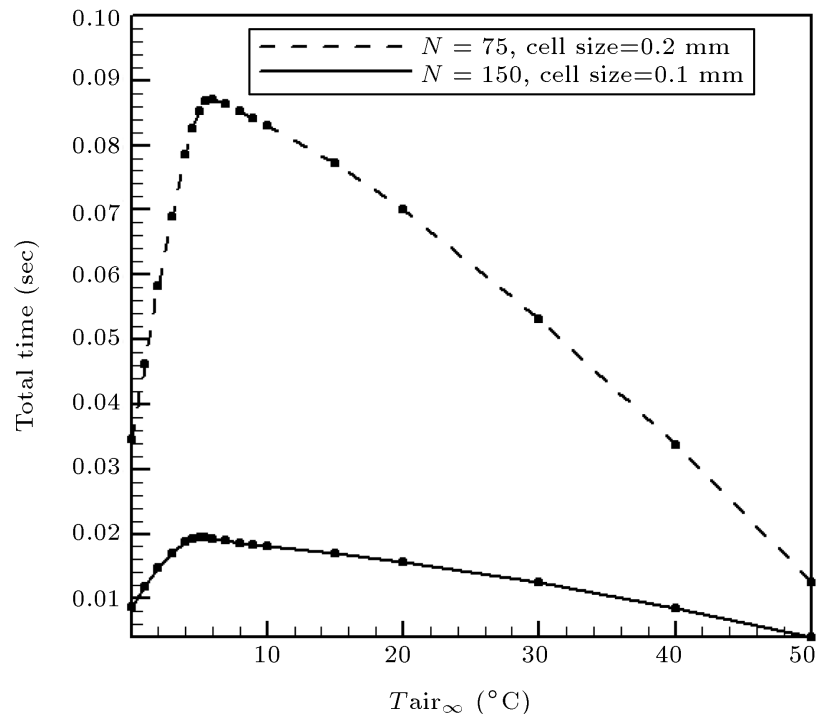

Figure 8. Total time needed to initiate vapor freezing in the nearest cells to the substrate surface versus the far-field air temperature for two different mesh sizes.

If $f=\frac{1}{8}$, then $T_{\text {sub, } \max } \cong-T_{\infty} / 18$; this result is in good agreement with that in Figure 7 for $T_{\infty}$ less than $15^{\circ} \mathrm{C}$. For larger $T_{\infty}$, the effect of $1^{\circ} \mathrm{C}$ subcooling of the vapor before condensation $\left(T=-1^{\circ} \mathrm{C}\right)$ and $N=150$ in Eq. (23) results in:

$$
T_{\text {sub }, \max } \cong-1-T_{\infty} /(150 f) \text {. }
$$

Thus, vapor subcooling would shift the result of Eq. (24) downward. Notice that since the variation in the size of the mesh from 0.2 to $0.1 \mathrm{~mm}$ results in a negligible variation in $T_{s u b, \max }$ (Figure 7 ), it can be concluded that the mesh size of $0.2 \mathrm{~mm}$ produces relatively accurate results.

The interesting and controversial point here is the freezing time depicted in Figure 8. According to this figure, time increases linearly by moving from the farfield temperature $\operatorname{Tair}_{\infty}=50^{\circ} \mathrm{C}$ to Tair $_{\infty}=30^{\circ} \mathrm{C}$ and, then, the time increment slope declines slowly. Thus, when the far-field temperature passes by about Tair $_{\infty}=5^{\circ} \mathrm{C}$, time declines steeply toward zero. But why? Time condensation and freezing of water vapor in the air is a function of two variables: far-field air temperature and water vapor content in the air. Of course, one cannot expect these two phenomena to be linear since the humidity ratio in the air is nonlinear. So far, at a far-field temperature close to zero degree in Celsius, vapor and the amount of water available for freezing are severely reduced. Therefore, the time required for condensation and freezing suddenly inclines toward zero. The behavior of the curve in Figure 8 can be similarly explained. In the start-up transient, the thickness of the thermal viscous layer increases as the square root of time elapses as follows:

$$
\delta=K \sqrt{t}
$$


Again, assume that the thermal viscous layer thickness is $\delta$, which has the value $\delta_{s s}$ in the steady state. This is because a perpendicularly impinging flow results in a viscous layer of constant thickness on the substrate. Thus, the initial thermal disturbance is similar to that of the semi-infinite solid with an initial uniform temperature whose surface temperature suddenly changes [33]. Eq. (26) can be obtained through the scaling analysis in the energy equation. For the present problem, this transient ceases when the thermal viscous layer achieves its steady-state value. The temperature distribution, which is taken to be linear for simplicity, in Eq. (22) results in the following by setting $y=\frac{\delta_{s s}}{N}$ as before and making use of Eq. (26):

$$
t=\left(\frac{\delta_{s s}}{N K}\right)^{2}\left(\frac{1}{f}\right)^{2}\left(\frac{T_{\infty}-T_{s u b}}{T-T_{s u b}}\right)^{2}, \quad t<t_{s s}
$$

where $\left(\frac{\delta_{s s}}{N K}\right)^{2}$ is constant. Given that the condensation begins at $T=0^{\circ} \mathrm{C}$ under no subcooling and at a substrate temperature of $T_{\text {sub }}=-1^{\circ} \mathrm{C}$, Eq. (27) becomes:

$$
t=\left(\frac{\delta_{s s}}{N K}\right)^{2}\left(\frac{1}{f}\right)^{2}\left(T_{\infty}+1\right)^{2}, \quad t<t_{s s} .
$$

Eq. (28) presents the parabolic increase of $t$ with increasing values of $T_{\infty}$, similar to the same trend shown in Figure 8, but with a constant value predicted after the thermal viscous layer is fully developed with a constant value of $\frac{1}{f}$. Of note, in the first region, $T$ air $_{\infty}<5^{\circ} \mathrm{C}$ in Figure 8, the factor $\left(\frac{1}{f}\right)^{2}$ is about 64 ; however, after this region, it tends to 9 . The effect of $0.5^{\circ} \mathrm{C}$ subcooling $\left(T=0.5^{\circ} \mathrm{C}\right)$ of the vapor before condensation and $N=150$ in Eq. (27) can lead to the following:

$$
t=4\left(\frac{\delta_{s s}}{N K}\right)^{2}\left(\frac{1}{f}\right)^{2}\left(T_{\infty}+1\right)^{2}, \quad t<t_{s s} .
$$

The prediction of Eq. (29) reveals that $0.5^{\circ} \mathrm{C}$ subcooling of the vapor before condensation increases the time lapse before condensation begins by a factor of 4 . Since factor $f$ is dependent on the amount of vapor in saturated gas, after the first region, a air $_{\infty}>5^{\circ} \mathrm{C}$, $\left(\frac{1}{f}\right)^{2}$ decreases faster than the increase of $\left(\frac{T_{\infty}-T_{s u b}}{T-T_{s u b}}\right)^{2}$; therefore, the total time decreases. Of note, in a physical experiment, condensation should immediately begin on the substrate surface; however, in numerical calculations, the size of the cell closest to the substrate controls the time at which condensation begins because the low temperature imposed by the substrate reaches the center of that cell by diffusion. Therefore, to demonstrate this process, computations are repeated with cell sizes of $0.01 \mathrm{~mm}$ and $0.02 \mathrm{~mm}$ since the time required for initializing condensation should vary as the inverse square of cell size, according to Eq. (29). Therefore, the results shown in Figure 8 show the varying trends at the freezing start time versus the far-field air temperature for two different mesh sizes. However, what will happen if the temperature of the substrate $\left(T_{\text {sub }}\right)$ is lower than that to start freezing $\left(T_{\text {sub, max }}\right)$ ?

In Figure 9(a), the results of substrate temperature $\left(T_{\text {sub }}\right)$ at $10^{\circ} \mathrm{C}$ lower than the maximum temperature of the substrate for the start of freezing $\left(T_{\text {sub,max }}\right)$ at a far-field temperature of $T$ air $_{\infty}=5^{\circ} \mathrm{C}$ are shown, and Figure 9(b) shows the same for $T$ air $_{\infty}=20^{\circ} \mathrm{C}$. As expected, the lower temperature substrate rises the

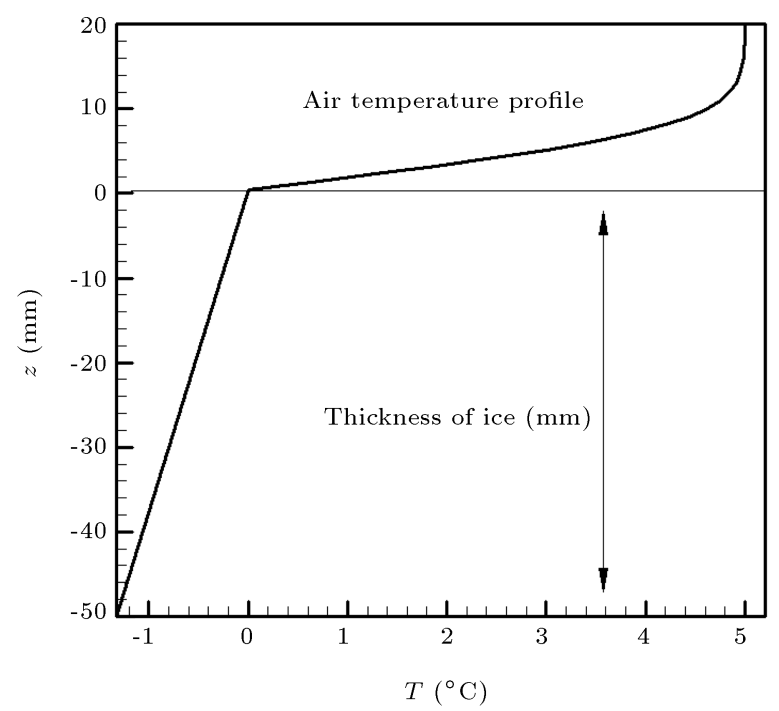

Figure 9a. Air and ice temperature profiles by reducing $10^{\circ} \mathrm{C}$ of the initial freezing temperature $\left(\right.$ Tair $_{\infty}=5^{\circ} \mathrm{C}$, $\left.T_{\text {sub }}=-10.32^{\circ} \mathrm{C}, T_{\text {sub }, \text { max }}=-0.32^{\circ} \mathrm{C}\right)$.

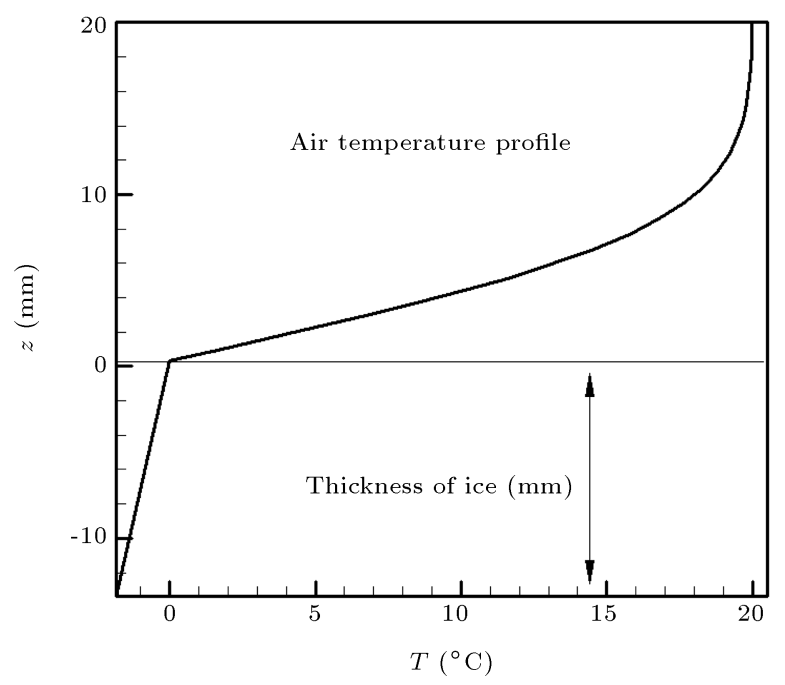

Figure 9 b. Air and ice temperature profiles by reducing $10^{\circ} \mathrm{C}$ of the initial freezing temperature $\left(\operatorname{Tair}_{\infty}=20^{\circ} \mathrm{C}\right.$, $\left.T_{\text {sub }}=-10.81^{\circ} \mathrm{C}, T_{\text {sub }, \text { max }}=-0.81^{\circ} \mathrm{C}\right)$. 
thickness of the ice. Moreover, a comparison between Figures $9(\mathrm{a})$ and $9(\mathrm{~b})$ shows that the same reduction of substrate temperature $\left(10^{\circ} \mathrm{C}\right)$ at the lower far-field temperature $\left(\operatorname{Tair}_{\infty}=5^{\circ} \mathrm{C}\right.$ ) exhibits a higher increase in the ultimate ice thickness (about $50 \mathrm{~mm}$ ) than the higher far-field temperature (Tair a $_{\infty}=20^{\circ} \mathrm{C}$ ), which is about $12 \mathrm{~mm}$.

\section{Conclusions}

The present paper investigated the saturated air water freezing in the two-dimensional stagnation flow on a flat plate. The air with a relative humidity of $100 \%$ vertically approached the cooled flat plate. At first, water vapor condenses and, then, if the substrate is cold enough, solidification occurs. The first important result is the highest substrate temperature $\left(T_{\text {sub,max }}\right)$, at which the saturated vapor turns into water and starts to freeze. These substrate temperatures were provided for different far-field air temperatures $\left(T\right.$ air $\left._{\infty}\right)$. In addition, the time required for freezing the first row of cells was presented. According to the obtained results, by increasing the far-field air temperature $\left(\right.$ Tair $\left._{\infty}\right)$ from $0^{\circ} \mathrm{C}$ to about $5^{\circ} \mathrm{C}$, the solidifying time increased; then, by increasing it further $\left(T_{\text {air }}^{\infty}\right)$, the solidifying time decreased. The temperature profiles, velocities in both directions, and water quantity changes in the cells were presented. Fluid temperature distribution and, most importantly, the ultimate frozen thickness for two different temperatures of far-field air and temperatures of the substrate were also presented. The charts with a substrate temperature below the freezing start temperature indicated that a $10^{\circ} \mathrm{C}$ drop in substrate temperature caused a large increase in the ultimate thickness of the ice for far-field air at Tair $_{\infty}=5^{\circ} \mathrm{C}$, while a drop of the same degree slightly increased the ultimate thickness of the ice for the air at Tair $_{\infty}=$ $20^{\circ} \mathrm{C}$.

\section{Nomenclature}

$\begin{array}{ll}a(t) & \text { Time-dependent flow strain rate } \\ \tilde{a} & \text { Dimensionless time-dependent strain } \\ & \text { rate } \\ a_{0} & \begin{array}{l}\text { Reference potential flow strain rate at } \\ \text { the start of time }\end{array} \\ A & \text { Area } \\ b_{1 \text { to } 5} & \text { Coefficient }\left({ }^{\circ} \mathrm{C}\right)^{0 \text { to }-4} \\ C & \text { Heat capacity coefficient } \\ E v a p & \text { Auxiliary variable } \\ H & \text { Latent enthalpy of water } \\ H_{l s} & 333.4 \mathrm{~kJ} / \mathrm{kg} \text { (liquid to solid) } \\ H_{v l} & 2501.0 \mathrm{~kJ} / \mathrm{kg} \text { (vapor to liquid) } \\ k & \text { Thermal conductivity }\end{array}$

$\begin{array}{ll}K & \text { Constant } \\ \dot{m} & \text { Mass of any participants } \\ N & \text { Mass flow rate } \\ p & \text { Number of nodes } \\ \tilde{p} & \text { Pressure } \\ \operatorname{Pr} & \text { Dimensionless pressure } \\ S, \dot{S}, \ddot{S} & \text { Prandtl number } \\ & \text { Thickness, velocity, and acceleration } \\ & \text { of ice evolution, respectively, in } z \\ \tilde{S}, \tilde{S} & \text { direction } \\ & \text { Dimensionless thickness and velocity } \\ & \text { of ice evolution, respectively, in } z \\ & \text { direction } \\ T & \text { Time } \\ T \text { air } & \text { Temperature } \\ T_{s u b, \text { max }} & \text { Temperature of air } \\ \forall & \text { The temperature of substrate for start } \\ u, w & \text { of freezing } \\ \tilde{u}, \tilde{w} & \text { Volume } \\ & \text { Velocity components near the plate in } \\ & x \text { and } z \text { directions } \\ & \text { near the plate in } x \text { and } z \text { directions } \\ & \text { Potential region velocity components } \\ & \text { in } x \text { and } z \text { directions } \\ & \text { Cartesian coordinates } \\ & \end{array}$

\section{Greek} $\delta$

$\delta^{*} \quad$ Displacement viscous layer thickness

$\zeta \quad$ Variable $(z-S(t))$

$\eta \quad$ Similarity variable

$\theta \quad$ Dimensionless temperature

$\nu \quad$ Kinematic viscosity

$\xi \quad$ Dimensionless $x$-axis

$\rho \quad$ Density

$\tau \quad$ Dimensionless time

$\Omega \quad$ Humidity ratio $=m_{\text {vapor }} / \dot{m}_{\text {air }}$

\section{Subscripts}

0

At the nearest cells of mesh to the ice (or first row of cells in fluid region)

$\infty \quad$ Free stream (far-field)

$i, k \quad$ Node (or cell) row and column numbers in $x$ and $z$ directions, respectively

$j \quad 1$ to 3 numbers (air, vapor, and water)

$l \quad$ Liquid phase (water)

ls Liquid to solid (phase change) 
$\max \quad$ The maximum substrate temperature that the first row of cells (or nearest cells to ice) can freeze

$\begin{array}{ll}N & \text { North } \\ S & \text { South } \\ s s & \text { Steady state } \\ \text { sub } & \text { Substrate plate } \\ \text { total } & \text { Total time of freezing the first row of } \\ & \text { cells } \\ v l & \text { Vapor to liquid (phase change) } \\ v & \text { Vapor }\end{array}$

\section{Superscripts}

$\begin{array}{ll}n & \text { Dimensionless } \\ n & \text { Timing step (old) } \\ n+1 & \text { Timing step (new) }\end{array}$

\section{References}

1. Stefan, J. "About the theory of ice formation, in particular on the ice formation in polar seas", [Uber die Theorie der Eisbildung, Insbesondere uber die Eisbildung in Polarmaere], A. Phys. Chem., 42, pp. 269-286 (1891).

2. Goodrich, L.E. "Efficient numerical technique for one dimensional thermal problems with phase change", Int. Journal of Heat Mass Transfer, 21, pp. 615-621 (1978).

3. Sparrow, E.M., Ramsey, J.W., and Harris, J.S. "The transition from natural convection controlled freezing to conduction controlled freezing", Journal of Heat Transfer, 103, pp. 7-13 (1983).

4. Lacroix, M. "Computation of heat transfer during melting of a pure substance from an isothermal wall", Numer. Heat Transfer B, 15, pp. 191-210 (1989).

5. Yeoh, G.H., Behnia, M., De Vahl Davis, G. et al. "A numerical study of three-dimensional natural convection during freezing of water", Int. J. Numer. Mech. Engng., 30, pp. 899-914 (1990).

6. Hadji, L. and Schell, M. "Interfacial pattern formation in the presence of solidification and thermal convection", Phy., Rev. A, 41, pp. 863-873 (1990).

7. Hanumanth, G.S. "Solidification in the presence of natural convection", Int. Comm. Heat Mass Transfer, 17, pp. 283-292 (1990).

8. Oldenburg, C.M. and Spera, F.J. "Hybrid model for solidification and convection", Numerical Heat Transfer, Part B: Fundamentals, 21(2), pp. 217-229 (1992).

9. Trapaga, G., Matthys, E.F., Valecia, J.J. et al. "Fluid flow, heat transfer and solidification of molten metal droplets impinging on substrates: comparison of numerical and experimental results", Metall. Trans. B, 23B, pp. 701-718 (1992).
10. Watanabe, T.I., Kuribayashi, and Honda, et al. "Deformation and solidification of a droplet on a cold substrate", Cham. Engng. 47, pp. 3059-3065 (1992).

11. Marchi, C. San, Liu, H., Lavernia, et al. "Numerical analysis of the deformation and solidification of a single droplet impinging on to a flat substrate", Journal Mater., 28, pp. 3313-3321 (1993).

12. Weidman, P.D. and Mahalingam, S. "Axisymmetric stagnation-point flow impinging on a transversely oscillating plate with suction", J. Engineering Mathematics, 31, pp. 305-318 (1997).

13. Shokrgozar Abbasi, A. and Rahimi, A.B. "Nonaxisymmetric three-dimensional stagnation-point flow and heat transfer on a flat plate", Trans. ASME J. Fluids Engineering, 131(7), pp. 074501.1-074501.5 (2009).

14. Shokrgozar Abbasi, A. and Rahimi, A.B. "Threedimensional stagnation-point flow and heat transfer on a flat plate with transpiration", J. Thermophys. Heat Transfer, 23(3), pp. 513-521 (2009).

15. Shafiq, A., Hammouch, Z. and Turab "Impact of radiation in a stagnation point flow of Walters' B fluid towards a Riga plate", Thermal Science and Engineering Progress, 6, pp. 33-37 (2018).

16. Hammouch, Z., Mekkaoui, T., and Sadki, H. "Similarity solutions of a steady MHD flow over a semiinfinite surface", Mathematics in Engineering, Science \& Aerospace (MESA), 8(1), pp. 109-117 (2017).

17. Eriksson, D., Gasch, T., and Malm, R. "Freezing of partially saturated air-entrained concrete: A multiphase description of the hygro-thermomechanical behavior", International Journal of Solids and Structures, 152-153, pp. 294-304 (2018).

18. Naganthran, K., Nazar, R., and Pop, I. "Unsteady stagnation-point flow and heat transfer of a special third grade fluid past a permeable stretching/shrinking sheet", Scientific Reports, 6(6), p. 24632 (2016).

19. Farooq, M., Javed, M., Ijaz Khan, M., et al. "Melting heat transfer and double stratification in stagnation flow of viscous nanofluid", Results in Physics, 7, pp. 2296-2301 (2017).

20. Hayat, T., Ijaz Khan, M., Waqas, M., et al. "Numerical simulation for melting heat transfer and radiation effects in stagnation point flow of carbon-water nanofluid", Computer Methods in Applied Mechanics and Engineering, 315, pp. 1011-1024 (2017).

21. Shokrgozar Abbasi, A., Rahimi, A.B. and Niazmand, H. "Exact solution of three-dimensional unsteady stagnation flow on a heated plate", J. Thermophys. Heat Transfer, 25(1), pp. 55-58 (2011).

22. Shokrgozar Abbasi, A. and Rahimi, A.B. "Investigation of two-dimensional unsteady stagnation flow and heat transfer impinging on an accelerated flat plate", Trans. ASME J. Heat Transfer, 134(6), pp. 064501.1064501.5 (2012). 
23. Shokrgozar Abbasi, A., Rahimi, A.B., and Mozayyeni, H. "Investigation of three-dimensional axisymmetric unsteady stagnation-point flow and heat transfer impinging on an accelerated flat plate", J. Applied Fluid Mechanics (JAFM), 9(1), Part 2, pp. 451-461 (2016).

24. Brattkus, K. and Davis, S.H. "Flow induced morphological instabilities: stagnation-point flows", Journal of Crystal Growth, 89, pp. 423-427 (1988).

25. Rangel, R.H. and Bian, X. "The inviscid stagnationflow solidification problem", Int. Journal of Heat Mass Transfer, 39(8), pp. 1591-1602 (1994).

26. Lambert, R.H. and Rangel, R.H. "Solidification of a supercooled liquid in stagnation-point flow", Int. Journal of Heat Mass Transfer, 46, pp. 4013-4021 (2003).

27. Yoo, J.S. "Effect of viscous plane stagnation flow on the freezing of fluid", Int. Journal of Heat and Fluid Flow, 1 21, pp. 735-739 (2000).

28. Mohammadiun, H., Amerian, V., Mohammadiun, M., et al. "Axisymmetric stagnation-point flow and heat transfer of nano-fluid impinging on a cylinder with constant wall heat flux", Thermal Science, 23(5B), pp. 3153-3164 (2019). DOI: 10.2298/TSCI171124090M (2018)

29. Rahimi, A.B. and Mozayeni, H. "Similarity solutions of axisymmetric stagnation-point flow and heat transfer of a viscous, boussinesq-related density fluid on a moving flat plate", Scientia Iranica, B, 21(4), pp. 1440-1450 (2014).

30. Alizadeh, R., Rahimi, A.B., and Najafi, M. "Nonaxisymmetric stagnation-point flow and heat transfer of a viscous fluid on a stationary cylinder", Scientia Iranica, 23(5), pp. 2238-2246 (2016).

31. Shokrgozar Abbasi, A. and Rahimi, A.B. "Solidification of two-dimensional viscous, incompressible stagnation flow", Int. Journal of Heat Transfer, 135, pp. 072301-8 (2013).
32. Shokrgozar Abbasi, A. "Three-dimensional axisymmetric solidification of a viscous incompressible flow in the stagnation point region", J. Applied Fluid Mechanics (JAFM), 10(1), Part 2, pp. 413-420 (2017).

33. Carslaw, H.S. and Jaeger, J.C., Conduction of Heat in Solids, 2nd Edn., Chapter 2, Oxford Science Publications (2000).

\section{Biographies}

Ali Shokrgozar Abbasi (1970) received his $\mathrm{PhD}$ from Mechanical Engineering Department, Ferdowsi University of Mashhad (2010) in Iran. The main field of his study is solidification in stagnation flow. During his $\mathrm{PhD}$ program, he established a three-dimensional computer program to predict the flow, temperature, and solidification of a fluid in stagnation flow. In 2011, he joined as an Assistant Professor to Payam Noor University of Mashhad, teaching with a greater emphasis on CFD, advanced heat transfer, advanced numerical calculations, and engineering mathematics. He works on techniques in computer programs of the modeling process in heat and fluid flow with phase change. There are also analytical and experimental methods in his works. His main interests include solidification, phase change to liquid, as well as heat and fluid flow. He has published about 10 ISI international journal papers and a book: "Convective heat transfer".

Mohsen Ghayeni (1990) obtained his BS degree in Mechanical Engineering from Birjand University Birjand, Iran, and is now a graduate student at the Mechanical Engineering Department at Payam Noor University in Mashhad. He is interested in freezing and melting, as well as heat and fluid flow. 